\title{
Modern DIB Surveys and DIB Environmental Behavior
}

\author{
P. Sonnentrucker \\ Space Telescope Science Institute, 3700 San Martin Drive Baltimore - MD21218, USA \\ email: sonnentr@stsci.edu
}

\begin{abstract}
Since the discovery of the Diffuse Interstellar Bands (DIBs) in the early 1920s, numerous research groups have taken on the task to obtain as complete a census of the DIBs as observationally possible in order to unravel characteristics that would allow the community to tie the DIB carriers to known species. Here, I will briefly review the efforts and progress that were made for the past two decades in producing new, deeper, and more complete DIB surveys. In particular, I will review the important role that these modern surveys are playing in revealing main observational characteristics of the DIBs and their dependence on the local physical conditions in the Galactic Interstellar Medium.
\end{abstract}

Keywords. ISM: clouds, ISM: structure, ISM: lines and bands, ISM: molecules, ISM: atoms

\section{Overview}

The Diffuse Interstellar Bands (DIBs) are weak absorption bands observed mainly in the 3900 to $9700 \AA$ wavelength range, though recent new detections in the near infrared extended their presence to about $2 \mu \mathrm{m}$ (see Geballe et al., this volume, and Cox et al., this volume). DIBs are ubiquitously detected in the interstellar medium (ISM) in the spectra of distant stars whether Galactic (see Herbig 1995, for a review) or extra-galactic (e.g., Welty et al. 2006; Cox et al. 2006; Lawton et al. 2008; Cordiner, this volume). In the Galaxy, the number of features identified as DIBs has grown from a few bands at the time of their discovery (Heger 1922) to over 500 bands today (e.g., Hobbs et al. 2008; 2009). The nature of the species producing the DIBs (called "DIB carriers") has been the subject of intense debate for over 90 years now. Speculation as to the nature of the candidate carriers has oscillated from gas phase species in the 1930s to solid-state entities in the 1950 s to 1970 s. However, spectroscopic evidence accumulated since the early $1980 \mathrm{~s}$ led to the now commonly accepted view that the DIB carriers are molecular in nature and mostly present in the gas phase in the Interstellar Medium (ISM; see Herbig 1995, for a review; Snow \& McCall 2006, for a review; Snow, this volume).

There are various indications that our knowledge of the ISM is quite incomplete, even though the physics and chemistry have been thought to be relatively straightforward (Federman, this volume; Liszt, this volume). There are numerous interstellar emission signatures from interstellar clouds (Unidentified Infrared Bands -UIBs; Allamandola et al. 1999, Extended Red Emission -ERE; Witt et al. 2006, unidentified sub-millimeter features; Muller et al. , this volume) and absorption features (the DIBs), believed to arise in molecules with a few common types of bonds, but without specific molecular identification to date (see Snow \& McCall 2006, for a review; Tielens 2008, for a review). The number of unknown species seems larger and of a more complicated nature than the $\sim 170$ species identified so far, pointing to the existence of a substantial reservoir of large molecules in interstellar space that are unidentified and therefore unaccounted for in theories of interstellar clouds and of star and planet formation. 
Our understanding of the distribution of atomic and molecular material in any given direction in the Galaxy remains rather unclear. Likewise, the structure of the ISM remains the subject of intense observational and theoretical studies to date (see Welty, this volume; Roueff, this volume; Federman, this volume). Figure 1 displays a schematic view of the main phases or cloud types that form the interstellar medium as we currently understand it. For each cloud type (or phase), the abundance of the main atomic or molecular tracers relative to hydrogen nuclei $\left(\mathrm{n}(\mathrm{X}) / \mathrm{n}_{H}\right)$ is plotted versus total hydrogen column density $(\mathrm{N}(\mathrm{H}))$. Also listed are the ranges in gas kinetic temperature $\left(\mathrm{T}_{\text {kin }}\right)$, in volume density of hydrogen nuclei $\mathrm{n}_{\mathrm{H}}$, in reddening $\mathrm{E}_{\mathrm{B}-\mathrm{V}}$, in percentage fraction of hydrogen nuclei in the form of molecular hydrogen $\mathrm{f}\left(\mathrm{H}_{2}\right)$, and a brief list of molecular tracers typically used in far-ultraviolet (FUV) and optical studies of the ISM. Note that there are no sharp boundaries between each cloud type (or phase) as they have been observed to overlap toward most Galactic sight lines (see Welty, this volume). As can be seen in Fig. 1, most of the atomic and molecular species used to probe the ISM physical conditions in the FUV and optical wavebands are present with varying abundances in cloud types (or multiple phases). In addition, most Galactic sight lines are comprised of a series of clouds with mixed physical conditions that are often spectrally unresolved (e.g., Sonnentrucker et al. 2003). As a result, most observational characterizations of the ISM structure along a given sight line contain various degrees of degeneracy that should be kept in mind when studying the behavior of the DIBs with respect to tracers of the ISM physical conditions.

Until the mid-70s and the development of solid state detectors, the study of variations in the DIBs equivalent width (EW) - a measure of the strength of the absorption - with varying local ISM physical conditions was technically limited to the four strongest known DIBs at 4430, 5780, 5797 and $6284 \AA$ (e.g. Josafatsson \& Snow 1987) even though a total of 39 DIBs had already been catalogued by Herbig (1975). With the subsequent arrival of high-resolution spectrographs along with detectors of greater sensitivity, the era of DIB systematic surveys was born with the pioneering works of Jenniskens \& Désert (1994) and Herbig (1995, for a review). Since the mid-90s, two major types of surveys have been routinely performed. The first type of survey focusses on observing a subset of the known DIBs toward targeted ISM environments with varying physical conditions. The second type of survey aims at obtaining as high a S/N DIB spectrum as technically possible, toward carefully-selected individual sight lines to obtain comprehensive census of DIBs for statistical studies of their properties. In this paper, I will briefly review what each type of survey taught us over the past 20 years about the main observational characteristics of the DIBs carriers and their dependence on the local physical conditions in the interstellar medium of our own Galaxy.

\section{Surveys of DIB subsets toward targeted ISM environments}

This type of approach has been by far the most commonly performed since the mid-70s. They aim at observing various subsets of DIBs - generally the strongest due to signal-tonoise and telescope time constraints - in various sample sizes (from 6 sight lines to 133 to-date) for sight lines carefully selected for their differences in gas physical conditions. The general goal of this type of research is to study the DIB response to their "local" environment in order to unravel cues to the nature of the DIB carriers, as DIB strengths and DIB strength ratios are known to vary significantly from sight line to sight line (see Krelowski, this volume). In the past 20 years, over 100 such surveys were published in which DIBs strength were compared with probes of the grain population and tracers 


\section{A Schematic View of Interstellar Medium}

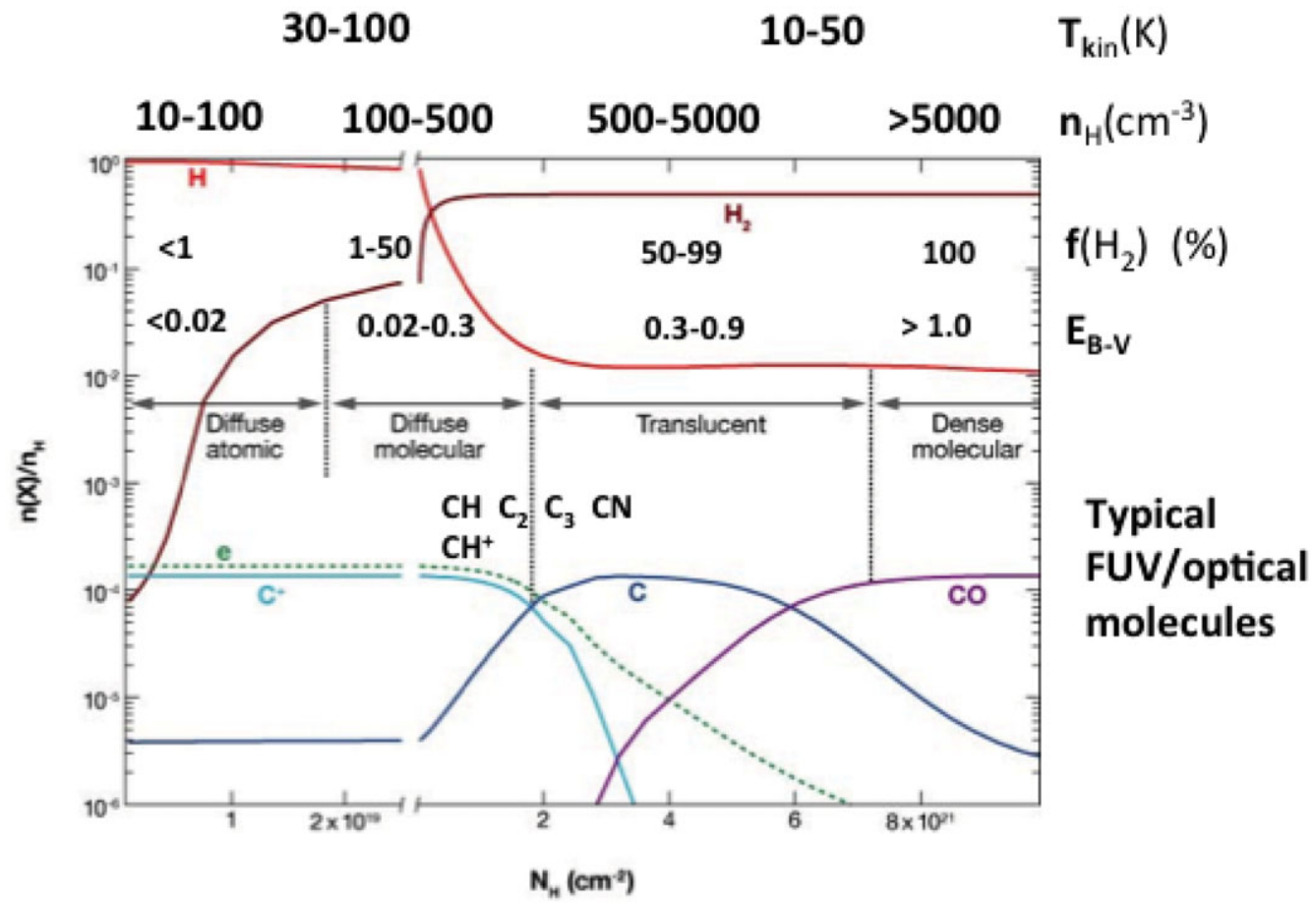

Figure 1. General schematic structure of the Interstellar Medium as seen using various far-ultraviolet (FUV) and optical diagnostics of the gas physical conditions. The abundance relative to hydrogen nuclei for the main tracers of each phase $\left(\mathrm{H}, \mathrm{H}_{2}, \mathrm{C}^{+}, \mathrm{C}, \mathrm{CO}\right)$ are plotted versus the total hydrogen column density, for each cloud type. Other typical FUV/optical tracers are listed in the figure. Note that their abundances are not reported on this figure. Representative values for the various parameters describing the cloud (or phase) physical conditions are also reported. Figure adapted from Snow \& McCall (2006) and Neufeld \& Wolfire (2009). The vertical dotted lines show model predictions of the transitions expected to occur between each phase. Observations, however, showed that the various clouds or phases overlap significantly toward most Galactic sight lines.

of the various ISM gas phases. Some of the findings resulting from these studies are highlighted below.

DIBs and interstellar dust distribution as measured by the reddening: $\left(\mathrm{E}_{B-V}\right)$

By the mid-90s, observations of the strongest DIBs $(4430,5780,5797,6284$ and $6613 \AA)$ showed an overall loose increase of the DIBs equivalent width with increasing reddening. The derived relationships showed significant scatter, in all cases, with departures from the mean by factors of at least three (see Herbig, 1995 for a review). This loose correlation between the reddening and DIB equivalent width along with its significant scatter have since been observed for all surveyed DIBs to-date regardless of their intrinsic strength. The recent observational confirmation that all known strong DIBs are detected at a $5 \sigma$ level toward sight lines with negligible reddening (e.g., Friedman et al. 2011), combined with the lack of polarization signatures in the profiles of the strongest DIBs (e.g., Cox et al. 2007) again add weight to the general consensus that most (if not all) DIB carriers are molecular in nature, are found in the gas phase and are well mixed with - but not necessarily related to - the dust component traced by the reddening in the Galactic ISM. 
DIBs and tracers of the atomic and molecular phases of the diffuse ISM

Herbig (1993) showed that a tight linear relationship existed between the equivalent width of the $5780 \AA \mathrm{DIB}$ and the column density of atomic hydrogen, $\mathrm{N}(\mathrm{H})$, while no significant correlation existed between that DIB and the column density of molecular hydrogen, $\mathrm{N}\left(\mathrm{H}_{2}\right)$, for the relatively small sample with overlapping DIB and $\mathrm{H}_{2}$ measurements available at that time. Krelowski et al. (1999) set to search for correlations between the 5780 and $5797 \AA$ DIBs with $\mathrm{CH}$, a surrogate tracer of $\mathrm{H}_{2}$ in diffuse molecular clouds (e.g. Sheffer et al. 2008) and with $\mathrm{CH}^{+}$, a presumed tracer of non-thermal processing (e.g. Godard et al. 2012) in an attempt to determine the phase in which these DIB carriers predominantly existed. They concluded, based on measurements in a sample of 70 Galactic sight lines, that the ratio of the $5797 \AA$ DIB to the $5780 \AA$ DIB showed no correlation with the column density of $\mathrm{CH}^{+}$per unit reddening. On the other hand, they found that this DIB ratio did exhibit a significant correlation with the column density of $\mathrm{CH}$ per unit reddening and concluded that the carriers of the 5797 and $5780 \AA$ DIBs were favored in - and traced- diffuse molecular and translucent type clouds.

With the launch of the Far Ultraviolet Spectroscopic Explorer (FUSE), direct measurements of $\mathrm{H}_{2}$ column densities were obtained during multiple Galactic surveys that greatly overlapped on-going DIB and atomic hydrogen surveys (e.g., Rachford et al. 2002; 2009). As a result, the DIB- $\mathrm{H}_{2}$ and DIB-H relationships were revisited by Friedman et al. (2011) toward a sample of 133 Galactic sight lines observed as part of a decade-long observing campaign aimed at constructing a large uniform DIB database using the Apache Point Observatory instrumentation (see York D.G. et al., this volume). The 5780-N(H) correlation reported by Herbig (1993) was extended to lower H column density sight lines than previously sampled and seven additional DIBs at 5797, 5705, 5487, 6196, 6204, 6283, 6613 $\AA$ were also found to exhibit tight relationships with $\mathrm{N}(\mathrm{H})$. For this subset of 8 DIBs that include the 5797 and $5780 \AA$ DIBs, the Friedman et al. survey sample exhibited loose correlations with the column density of $\mathrm{H}_{2}$ for $\mathrm{N}\left(\mathrm{H}_{2}\right)>10^{20} \mathrm{~cm}^{-2}$ (or $\mathrm{N}(\mathrm{CH})>$ few $\times 10^{12} \mathrm{~cm}^{-2}$ ), as suggested by the Krełowksi et al. study. However, the latter correlations break down for $\mathrm{N}\left(\mathrm{H}_{2}\right)<10^{20} \mathrm{~cm}^{-2}$, a regime not probed by the former study. The Friedman et al. survey, hence, suggests that the correlation between $\mathrm{N}\left(\mathrm{H}_{2}\right)$ and the strength of the $5797,5705,5487,6196,6204,6283,6613 \AA$ DIBs is mostly driven by the general correlation of the DIB strength with total hydrogen column density rather than by a specific correlation with $\mathrm{H}_{2}$, a conclusion already derived by Herbig (1993) for the $5780 \AA$ DIB.

Using the same survey data as Friedman et al., Thorburn et al. (2003) reported on the existence of a subset of DIBs that showed to increase in strength with increasing column density of molecular carbon per unit reddening $\left(\mathrm{N}_{(}\left(\mathrm{C}_{2}\right) / \mathrm{E}_{B-V}\right)$. These DIBs were subsequently named the $\mathrm{C}_{2}$-DIBs. Molecular carbon is a second generation molecule that results from the chemical interaction between $\mathrm{C}^{+}$and $\mathrm{H}_{2}$ (e.g., Federman et al. 1994). As a result, $\mathrm{C}_{2}$ traces a much denser gas-phase regime than does atomic hydrogen. The Thorburn et al. (2003) findings, hence, revealed the presence of a subset of DIB carriers that are favored in diffuse molecular clouds or translucent clouds where larger volume densities and greater shielding from the average background Galactic UV radiation field are measured compared to those typically measured in diffuse atomic clouds (see Fig. 1). DIBs, Galactic FUV radiation and ionization

DIBs were known to vary significantly in strength since the mid-70 (e.g. Herbig 1975). A search for the strongest known DIBs toward dense dark clouds revealed that the DIBs were weak or completely absent at the cloud cores but grew in strength toward the cloud edges. This phenomenon, subsequently called the "skin" or "edge" effect (Snow \& Cohen 1974; see Snow, this volume) was since unequivocally observed toward the sight line to 
the background star HD 62542. This sight line is known to contain a single molecular core that lost its diffuse gas envelope due to stripping by stellar winds and radiative pressure (Cardelli et al. 1990). Recent observations of HD 62542 by Snow et al. (2002) and Ádámkovics et al. (2005) revealed that the DIBs that correlate well with atomic hydrogen are extremely weak (or absent) compared to typical Galactic sight lines while 10 of the $18 \mathrm{C}_{2}$-DIBs are present. This result again strongly suggests the existence of a bi-modal distribution among the DIB carriers with many of the carriers favored in diffuse atomic gas and some carriers favored in denser gas with higher molecular fractions. These observed differences might point toward intrinsic differences in the molecular nature of the carriers of these 2 sets of DIBs. They might also be the result of changes in the DIB carrier's structure that could be triggered by variations in local gas physical processes (ionization, neutralization, hydrogenation) for a given DIB population.

DIBs were observed to be weaker by factors of 2 or more per unit reddening in photondominated regions (PDR, see Jenniskens, Ehrenfrend \& Foing 1994). In a subsequent survey of 38 Galactic sight lines of varying local physical conditions Sonnentrucker et al. (1997) quantified the variation in strength with reddening of the 5780, 5797, 6379 and $6613 \AA$ A DIBs and revealed the existence of a different "sweet spot" where each DIB strength peaked. These variations were interpreted as being most likely due to a change in the DIB carrier's charge state. The latter study along with that of Cami et al. (1997) established in a quantitative manner that DIB carriers indeed responded differently to exposure to local FUV radiation conditions (see Foing, this volume). Gnaciński et al. (2007) looked for potential relationships between the strength of 8 DIBs including those studied by Sonnentrucker et al. (1997) and the electron volume density $\left(\mathrm{n}_{e}\right)$ as measured from the column density ratio of singly-ionized magnesium to neutral magnesium (Mg II/ $\mathrm{MgI}$ ) for a sample of 11 sight lines. Using this particular tracer, they found no significant correlation between $\mathrm{n}_{e}$ and the strength of these DIBs and concluded that the carriers of these DIBs did not seem to exhibit a change in charge state either because of the probed range $\left(0.009<\mathrm{n}_{e}<2.5 \mathrm{~cm}^{-3}\right)$ or because the carriers of these DIBs were favored in a gas phase not traced by $\mathrm{Mg}$. These types of approaches are important as they attempt to quantitatively link the measured DIB behavior with measurable tracers of the physical conditions in the gas harboring the DIB carriers and should be extended to a larger set of DIBs toward a greater sample of sight lines, on the one hand, and a larger set of tracers of the electron density (e.g., Sonnentrucker et al. 2003), on the other hand.

DIBs profiles and temperature tracers of the ISM gas

With the development of high spectral resolution instrumentation, the asymmetries observed in the profile of some of the strongest DIB were resolved into double- or triplepeaked substructures. The most notorious and most studied case involves the triplepeaked substructure resolved in the profile of the $6613 \AA$ DIB (see Sarre, in this volume). The origin of these substructures can be two-fold. These substructures can be induced by blends of DIB absorption arising in discrete clouds along the probed sight lines. In such cases, one has a rare opportunity to constrain the DIB carrier's response to the local ISM conditions in individual clouds (e.g., Ruiterkamp et al. 2005). These substructures can also be intrinsic to the DIB profile and reveal the rotational structure of the molecule within a given electronic state. In such a case, the separation between each sub-feature can be used to constrain the structure of the molecule (e.g., Sarre et al. 1995; Sarre, this volume; Oka et al., this volume).

Numerous studies have now shown that the multiple substructures observed ubiquitously in the profile of the $6613 \AA$ are intrinsic in nature. Sonnentrucker et al. (1999) observed the latter DIB in sample of 7 sight lines selected to probe a $50 \mathrm{~K}$ range in gas kinetic temperature derived from $\mathrm{H}_{2}$ measurements in an attempt to find an increase 
Table 1. Deep DIB Surveys Since the Mid-90s. Column (1) lists the sight line targeted by each study. Column (2) gives the wavelength coverage of each survey. Columns (3) \& (4) list the detection limits for the DIB strength (EW) and minimum DIB bandwidth (FWHM), respectively. Column (5) reports the sight line reddening. Column (6) reports the number of DIBs listed in each survey, and Column (7) lists the reference for each study.

\begin{tabular}{|c|c|c|c|c|c|c|}
\hline $\begin{array}{c}\text { Sight line } \\
\text { (SpT) }\end{array}$ & $\begin{array}{c}\text { Coverage } \\
{[\AA]}\end{array}$ & $\begin{array}{c}\mathbf{E W}_{\min } \\
{[\mathrm{m} \AA]}\end{array}$ & $\begin{array}{c}\text { FWHM } \\
{[\AA]}\end{array}$ & $\mathbf{E}_{B-V}$ & $\begin{array}{c}\text { DIB } \\
\text { Number }\end{array}$ & Reference $^{*}$ \\
\hline HD 183143 & $3900-13300$ & 4 & 0.8 & 1.27 & 127 & 1,2 \\
BD+63 1964 & $3900-6812$ & 4 & 0.5 & 1.01 & 226 & 3 \\
HD 23180 & $4460-8800$ & $\ldots$ & $\ldots$ & 0.26 & 271 & 4 \\
$\sigma-\zeta$ sight lines & $5650-6865$ & 3 & $\ldots$ & 0.14 & 213 & 5 \\
HD 204827 & $3900-8100$ & 1 & 0.2 & 1.11 & 380 & 6 \\
HD 183143 & $3900-8100$ & 1 & 0.2 & 1.27 & 414 & 7 \\
\hline
\end{tabular}

* (1) Herbig 1995; (2) Jenniskens \& Désert (1994); (3) O'Tuarisg et al. (2000); (4) Galazutdinov et al. (2000); (5) Weselak et al. (2000); (6) Hobbs et al. (2008) and (7) Hobbs et al. (2009).

in the profile triple-peak separation and constrain the DIB carrier's structure. No such variations in the substructure positions were found leading the authors to conclude that either the gas kinetic temperature derived from $\mathrm{H}_{2}$ was not an appropriate probe of the rotational structure for this particular DIB carrier or that the dynamical range in temperature used for that particular study was insufficient. Subsequent work by Cami et al. (2004) and Friedman et al. (2011), seem to suggest that the gas temperature derived from $\mathrm{H}_{2}$ measurements is, indeed, not a sensitive probe of the rotational structure for this particular DIB carrier. Kaźmierczak et al. (2009) searched for a similar behavior within the double-peaked profile of the $6196 \AA$ DIB using a sample of 11 sight lines with known molecular carbon $\left(\mathrm{C}_{2}\right)$ detections. They found a weak correlation between the DIB full-width-at half-maximum (FWHM) and the gas excitation temperature calculated from the lowest rotational levels of $\mathrm{C}_{2}$. They concluded that the $6196 \AA$ AIB seemed to respond to excitation conditions similar to those traced by $\mathrm{C}_{2}$ but also emphasized the need for a larger target sample to verify the trend suggested by their current dataset.

\section{Deep surveys of DIBs toward individual sight lines}

While surveys of DIB subsets throughout the Galactic disk are instrumental at constraining the response of the DIB carriers to their "local" environment and at revealing unusual sight lines (see York D. G. et al., this volume), these surveys are not designed to give an unbiased census of the DIB spectrum. As complete a list of DIBs as feasible, is nevertheless warranted to allow for detailed comparisons between existing observations and current laboratory spectra (e.g., Salama et al. 2011). These deep surveys are also needed to perform unbiased statistical studies of the distribution of DIBs bandwidth (FWHM), DIBs strength (EW) and DIB spacing in an attempt to put some constraints on the identity of the DIB carriers. However, pushing the detection limit for the DIBs in the optical requires substantial observing time, ideally with the same or similar facilities to reduce the impact of systematics when detecting new weak or broad features (see York B. et al., this volume). As a result, only 7 deep DIB surveys have been published to date.

Table 1 summarizes the main observational characteristics of each survey and shows that five of the seven surveys exhibit very similar detection limits in DIB equivalent

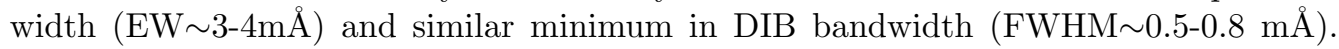
The number of reported DIBs increased by about a factor of 2 between the first systematic surveys of DIBs published by Jenniskens \& Désert (1994) and Herbig (1995) and the 

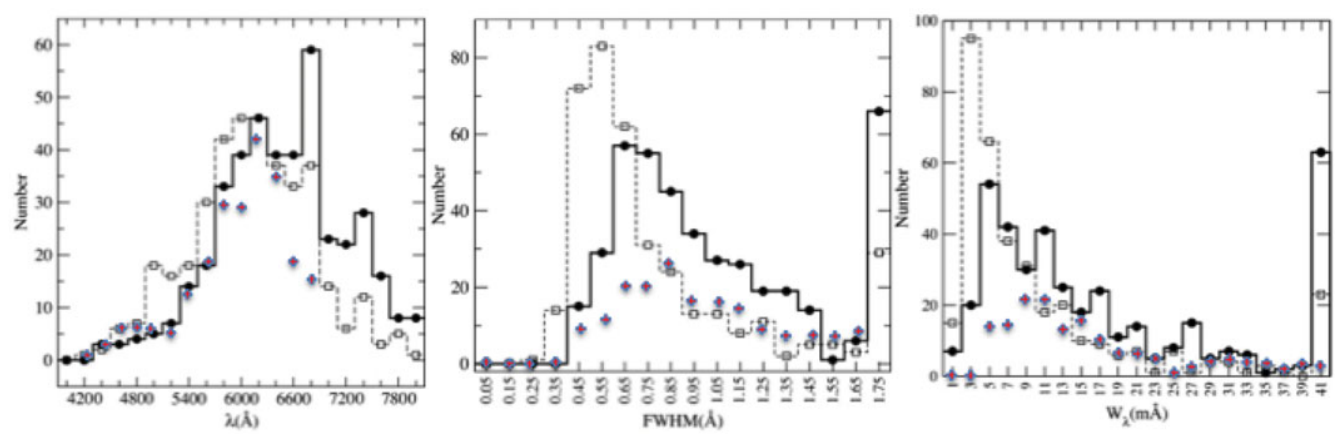

Figure 2. Distribution of the newly detected DIBs toward HD 183143 (filled circles), HD 204827 (open squares) and BD+631964 (red crosses) by wavelength (left), by bandwidth (middle) and by equivalent width (right). Note that both the depth and the wavelength coverage of the BD+63 1964 survey are lower than those for the other 2 sight lines (see Table 1 for details).

surveys performed in the early 2000 . These 5 studies reveal that about $70 \%$ of the newly reported DIBs are detected in the range 4900-6890 $\AA$, regardless of the sight lines reddening, again emphasizing the gas-phase nature of the DIB carriers. With an improvement of the detection limit by a factor of $\sim 3$ in both the DIB strength and minimum DIB bandwidth, Hobbs et al. $(2008,2009)$ increased the number of newly detected DIBs yet again by about a factor of 2 , bringing the total number of known DIB to over 500 features currently. Figure 2 compares the distribution of the number of newly detected DIBs by wavelength ( $\lambda$, left panel), DIB bandwidth (FWHM, middle panel) and DIB strength ("W", right panel) toward the 3 sight lines for which detection limits for both the DIB strength and the DIB bandwidths were quoted in the published work. These sight lines are HD 183143 (Hobbs et al. 2009, black filled circles), HD 204827 (Hobbs et al. 2008, open squares) and BD+631964 (O'Tuairisg et al. 2000, red crosses).

Differences between HD 204827 and HD 183143 are notable for the 3 distributions and have been discussed in Hobbs et al. (2009). Briefly, their surveys showed that the distribution in wavelength is overall redder toward HD 183143 than toward HD 204827. For FWHM $>0.7 \AA$ (a regime where sight line variations in velocity distribution among absorbing clouds becomes unimportant) the DIB bandwidth distribution is generally broader toward HD 183143 than toward HD 204827. When comparing their DIB strength distributions both sight lines show a steady decrease of the DIB number above an equivalent width of about $5 \mathrm{~m} \AA$ indicating that, in both cases, weak DIBs dominate the overall distribution when detected. However, HD 204827 exhibits a significantly larger number of weak DIBs than HD 183143 which might mostly reflect the difference in their DIB bandwidth distribution as the selectively narrower DIB distribution observed toward HD 204827 favors detection of weaker DIBs at equal spectral quality. To date, HD 204827 remains the sight line with the highest measured column density of $\mathrm{C}_{2}$ and the strongest measured $\mathrm{C}_{2}$ DIBs (see Thorburn et al. 2003). Toward HD 183143, on the other hand, no detections of $\mathrm{C}_{2}$ lines or $\mathrm{C}_{2}$ DIBs were reported by Hobbs et al. (2009). Hence, the significant differences in DIB distributions noticed between these 2 sight lines point toward clear differences either in the DIB carrier populations themselves or in the DIB carriers response to the known differences in gas physical conditions observed between the 2 sight lines.

When comparing the BD+63 1964 survey with the Hobbs $(2008,2009)$ surveys, over the range in wavelength common to the 3 sight lines, one can see that the distribution of DIBs by wavelength toward $\mathrm{BD}+631964$ seems more similar to that toward HD 183143 than 
the distribution toward HD 204827. The drop in DIB number beyond $6600 \AA$ might not be significant for BD+631964 considering the cut-off of the O'Tuairisg survey. However, BD+63 1964 exhibits both a DIB bandwidth distribution (FWHM > $0.7 \AA$, middle) and a DIB strength distribution ( $\mathrm{W}>9 \mathrm{~m} \AA$, right) that are very similar to those observed toward HD 204827. Such similarities might indicate that these sight lines exhibit very similar populations of DIB carriers over the range common to both surveys or have very similar gas physical conditions. As mentioned earlier, HD 204827 has the highest measured column density of $\mathrm{C}_{2}$ and, thus, the strongest $\mathrm{C}_{2}$ DIBs. It would be most interesting to perform as deep a survey toward BD+631964 as Hobbs et al. obtained toward HD 204827 in order to establish the nature of DIB distribution in width below $0.7 \AA$ and DIB strength below $9 \mathrm{~m} \AA$, for direct comparisons with Hobbs et al. (2008) and to search for $\mathrm{C}_{2}$ DIBs. The weakness of this sub-class of DIBs would then suggest that the carrier population producing the broad (FWHM $>0.9 \AA)$ and strong $(\mathrm{W}>11$ $\mathrm{mA}$ ) DIBs are extremely similar toward both sight lines.

A search for specific spacing between adjacent DIBs was also performed by O'Tuairisg et al. (2000) and Hobbs et al. $(2008,2009)$ in order to uncover preferred patterns that would be indicative of a particular molecular structure. Using the total number of possible pairs of DIBs detected toward HD 183143 and HD 204827 whose wavenumber splittings did not exceed $400 \mathrm{~cm}^{-1}(\sim 2.5 \mathrm{m \AA})$, Hobbs et al. found no preferred DIB spacings in either of the 2 sight lines. They also found that the distribution of gaps between adjacent DIBs were essentially random for both sight lines too. Similar conclusions were drawn by O'Tuairisg et al. (2000) toward the sight line to BD+631964.

\section{Conclusions}

Over the past 20 years, both types of survey have revealed that a large number of DIBs are very well correlated with the column density of atomic hydrogen but poorly correlated with the column density of molecular hydrogen, hence, indicating that their carriers are favored in atomic gas clouds (or phase) of low molecular fractions. On the other hand, a small sub-set of DIBs was discovered to be enhanced in sight lines with significant column densities of molecular carbon per unit reddening, hence, revealing the presence of a set of DIB carriers favored in denser gas clouds. While the nature of these DIB carriers remains unknown, these surveys have demonstrated that certain subsets of DIBs can be used as diagnostic tracers of the various ISM phases often mixed and partially unresolved along typical Galactic sight lines. The few deep DIB surveys published to date have revealed no particular patterns that would be reminiscent of a particular molecule structure in the DIB distributions. These surveys however showed that the DIB distributions in bandwidth and band strength tend to be dominated by weak DIBs whenever detectable. None of the existing surveys is complete in detecting broad DIBs $($ FWHM $>6 \AA$ ), though. The existence of these broad DIBs has been suggested but systematic searches for such features have only recently been initiated (see York B. et al., this volume).

\section{Acknowledgements}

I wish to thank the organizers for their invitation and for a highly stimulating and productive conference. Part of the work presented here is the result of an ongoing collaboration with Don York, Lew Hobbs, Dan Welty, Scott Friedman, Julie Dahlstrom, Ted Snow, Brian Rachford and Brian York. 


\section{References}

Ádámkovics, M., Blake, G. A., \& McCall, B. J. 2005 ApJ, 625, 857

Allamandola, L. J., Hudgins, D. M., \& Sandford, S. A. 1999 ApJL, 511, 115

Cami, J., Sonnentrucker, P., Ehrenfreund, P., \& Foing, B. H. 1997, A\&A, 326, 822

Cami, J., Salama, F., Jiménez-Vicente, J., Galazutdinov, G. A., \& Krełowski, J. 2004, ApJ, 611, 113

Cardelli, J. A., Edgar, R. J., Savage, B. D., \& Suntzeff, N. B. 1990, ApJ, 362, 551

Cox, N. L. J., Cordiner, M. A., Cami, J., Foing, B. H., Sarre, P. J., Kaper, L., \& Ehrenfreund, P. 2006, A\&A, 447, 991

Cox, N. L. J., Boudin, N., Foing, B. H., Schnerr, R. S., Kaper, L., Neiner, C., Henrichs, H., Donati, J.-F., \& Ehrenfreund, P. 2007, A\&A A, 465, 899

Federman, S. R., Strom, C. J., Lambert, D. L., Cardelli, J. A., Smith, V. V., \& Joseph, C. L. 1994, ApJ, 424, 772

Friedman, S. D., York, D. G., McCall, B. J., Dahlstrom, J., Sonnentrucker, P., Welty, D. E., Drosback, M. M., Hobbs, L. M., Rachford, B. L., \& Snow, T. P. 2011, ApJ, 727, 33

Galazutdinov, G. A., Musaev, F. A., Krełowski, J., \& Walker, G. A. H. 2000, PASP, 112, 648

Gnaciński, P., Sikorski, J. K., \& Galazutdinov, G. A. 2007, A\&A A, 469, 201

Godard, B., Falgarone, E., Gerin, M., Lis, D. C., De Luca, M., Black, J. H., Goicoechea, J. R., Cernicharo, J., Neufeld, D. A., Menten, K. M., \& Emprechtinger, M. 2012, A\& $A$, 540, 87

Herbig, G. H. 1975, ApJ, 196, 129

Herbig, G. H. 1993, ApJ, 407, 142

Herbig, G. H. 1995, ARAA, 33, 19

Hobbs, L. M., York, D. G., Snow, T. P., Oka, T., Thorburn, J. A., Bishof, M., Friedman, S. D., McCall, B. J., Rachford, B., Sonnentrucker, P., \& Welty, D. E. 2008, ApJ, 680, 1256

Hobbs, L. M., York, D. G., Thorburn, J. A., Snow, T. P., Bishof, M., Friedman, S. D., McCall, B. J., Oka, T., Rachford, B., Sonnentrucker, P., \& Welty, D. E. 2009, ApJ, 705, 32

Jenniskens, K. \& Désert, F.-X 1994, A\&AS, 106, 39

Jenniskens, K., Ehrenfreund, P., \& Foing, B. H. 1994, A\&A, 281, 517

Josafatsson, P. \& Snow, T. P. 1987, ApJ, 321, 952

Kaźmierczak, M., Gnaciński, P., Schmidt, M. R., Galazutdinov, G., Bondar, A., \& Krełowski, J. 2009, $A \& A, 498,785$

Krełowski, J., Ehrenfreund, P., Foing, B. H., Snow, T. P., Weselak, T., Tuairisg, S.Ó., Galazutdinov, G. A., \& Musaev, F. A. 1999, A\& A, 347, 235

Lawton, B., Churchill, C. W., York, B. A., Ellison, S. L., Snow, T. P., Johnson, R. A., Ryan, S. G., \& Benn, C. R. 2008, AJ, 136, 994

Neufeld, D. A. \& Wolfire, M. 2009, ApJ, 706, 1594

Rachford, B. L., Snow, T. P., Tumlinson, J., Shull, J. M., et al. 2002, ApJ, 577, 221

Rachford, B. L., Snow, T. P., Destree, J. D., et al. 2009, ApJS, 180, 125

Ruiterkamp, R., Cox, N. L. J., Spaans, M., Kaper, L., Foing, B. H., Salama, F., \& Ehrenfreund, P. $2005, A \mathscr{E} A, 432,515$

Salama, F., Galazutdinov, G. A., Krełowski, J., Biennier, L., Beletsky, Y., \& Song, In-Ok 2011, ApJ, 728, 154

Sarre, P. J., Miles, J. R., Kerr, T. H., Hibbins, R. E., Fossey, S. J., \& Somerville, W. B. 1995, MNRAS, 277, 41

Sheffer, Y., Rogers, M., Federman, S. R., Abel, N. P., Gredel, R., Lambert, D. L., \& Shaw, G. 2008, ApJ, 687, 1075

Snow, T. P. \& Cohen, J. G. 1974, ApJ, 194, 313

Snow, T. P. \& McCall, B. J. 2006, ARAA, 44, 367

Snow, T. P., Welty, D. E., Thorburn, J., Hobbs, L. M., McCall, B. J., Sonnentrucker, P., \& York, D. G. 2002, ApJ, 573, 670

Sonnentrucker, P., Cami, J., Ehrenfreund, P., \& Foing, B. H. 1997, A\&AA, 327, 1215

Sonnentrucker, P., Foing, B. H., Breitfellner., M., \& Ehrenfreund, P. 1999, A\& A, 346, 936

Sonnentrucker, P., Friedman, S. D., Welty, D. E., York, D. G., \& Snow, T. P. 2003, ApJ, 596, 350 
Thorburn, J. A., Hobbs, L. M., McCall, B. J., Oka, T., Welty, D. E., Friedman, S. D., Snow, T. P., Sonnentrucker, P., \& York, D. G. 2003, ApJ, 584, 339

Tielens, A. G. G. M. 2008, ARAA, 46, 289

Tuairisg, S.Ó., Cami, J., Foing, B. H., Sonnentrucker, P., \& Ehrenfreund, P. 2000, A\&ऽAS, 142, 225

Welty, D. E., Federman, S. R., Gredel, R., Thorburn, J. A., \& Lambert, D. L. 2006, ApJS, 165, 138

Weselak, T., Schmidt, M., \& Krełowski, J. 2000, A\&AS, 142, 239

Witt, A. N., Gordon, K. D., Vijh, U. P., Sell, P. H., Smith, T. L., \& Xie, R.-H. 2006, ApJ, 636, 303 